\title{
Implicações da avaliação geriátrica ampla na qualidade de vida em pessoas idosas com câncer: uma revisão integrativa
}

\author{
Implications of comprehensive geriatric assessment on quality of life in older adults with cancer: an \\ integrative review
}

Valéria Sgnaolini,2 ID

Vanessa Sgnaolin' ID

Rodolfo Herberto Schneiderl $\mathbb{D}$

\section{Resumo}

A avaliação geriátrica ampla (AGA) melhora a qualidade do cuidado das pessoas idosas com câncer, pois permite a identificação de problemas geriátricos e fragilidades que tenham implicações na saúde do indivíduo. Apesar dos benefícios da AGA, dificuldades relacionadas ao tempo e gastos com essa ferramenta limitam sua implantação na prática. O objetivo desta revisão é avaliar a relação entre a AGA e a qualidade de vida (QV) de pessoas idosas com câncer, através de uma revisão integrativa da literatura. Foi realizada uma busca por artigos nas bases de dados PubMed, MEDLINE, IBECS e LILACS, publicados entre 2015 e 2020, que abordassem as implicações da AGA na QV de pessoas idosas com câncer e, dos 298 estudos encontrados, 21 foram selecionados para análise. Esses demonstraram que a AGA desempenha função importante ao identificar pessoas idosas com maior risco de comprometimento da QV durante a evolução da neoplasia e do tratamento oncológico, bem como ao orientar a indicação de intervenções geriátricas específicas que previnam a deterioração da QV. Assim, a presente revisão destaca a importância da avaliação integral das pessoas idosas com câncer que, através de diferentes âmbitos, sejam prognósticos ou intervencionistas, desenvolve um papel fundamental na preservação da QV dessa população. Compreende-se a necessidade de desenvolver estratégias para incorporação da AGA no cuidado das pessoas idosas com câncer.

\section{Abstract}

Comprehensive geriatric assessment (CGA) improves the quality of care for older adults with cancer, as it identifies geriatric problems and weaknesses that have implications for the health of the individual. Despite the benefits of CGA, difficulties related to time of application and cost of this tool limit its implementation in practice. The purpose of

Palavras-chave: Avaliação Geriátrica. Neoplasias. Qualidade de vida.
Keywords: Geriatric Assessment. Neoplasms. Quality of Life.

\footnotetext{
Pontifícia Universidade Católica do Rio Grande do Sul (PUCRS), Escola de Medicina, Programa de Pós-Graduação em Gerontologia Biomédica. Porto Alegre, RS, Brasil.

2 Hospital Mãe de Deus, Centro Integrado de Oncologia. Porto Alegre, RS, Brasil.
}

Os autores declaram não haver conflito na concepção deste trabalho.

Não houve financiamento para a execução deste trabalho. 
this review is to evaluate the relationship between CGA and the quality of life (QoL) of older adults with cancer, through an integrative review. A search was performed for articles in the PubMed, Medline, IBECS and Lilacs databases, published between 2015 and 2020, that addressed the implications of CGA on the QoL of older adults with cancer and, of the 298 studies found, 21 were selected for analysis. These studies revealed that CGA performs an important role in identifying older adults with a higher risk of QoL impairment during the course of cancer and cancer treatment, as well as guiding the indication of specific geriatric interventions that prevent the deterioration of QoL. Thus, the present review highlights the importance of the broad assessment of older adults with cancer, which, through different spheres, whether prognostic or interventionist, can play a fundamental role in preserving the QoL of this population. It is imperative that strategies are developed that incorporate CGA in the care of older adults with cancer.

\section{INTRODUÇÃO}

O câncer é uma doença associada com o envelhecimento e um importante problema de saúde pública, pois representa atualmente a segunda causa de óbitos no mundo, com tendência de crescimento nos próximos anos ${ }^{1,2}$. O atendimento da pessoa idosa com câncer é muitas vezes desafiador, devido à sua constelação complexa de questões médicas e psicossociais, sendo necessário o trabalho conjunto de uma equipe interdisciplinar, a fim de garantir o cuidado integral desse paciente ${ }^{3,4}$. No entanto, como existe uma grande heterogeneidade no estado de saúde de pessoas idosas de idade semelhante, é importante identificar os indivíduos que apresentem fatores de risco que possam influenciar negativamente a evolução e tratamento oncológico ${ }^{5,6}$.

Uma ferramenta útil na gestão e acompanhamento das pessoas idosas com câncer é a avaliação geriátrica ampla (AGA), um processo de diagnóstico multidimensional, que vai além da idade cronológica, para avaliar de forma abrangente o estado de saúde ${ }^{7,8}$. Consiste em uma abordagem sistemática, com ênfase em parâmetros funcionais, cognitivos, nutricionais, psicológicos e socioambientais, além da identificação de comorbidades e medicamentos utilizados ${ }^{9,10}$.

A AGA permite a identificação de problemas geriátricos e fragilidades que tenham implicações na saúde do indivíduo e demonstrou ser um marcador preditivo para sobrevida e tolerância ao tratamento em pessoas idosas com câncer ${ }^{3,10,11}$. Além disso, fornece uma plataforma para lidar com as necessidades individualizadas e manejar condições reversíveis, criando oportunidades para melhorar o estado funcional de pessoas idosas com câncer e auxiliar na elaboração de um plano geriátrico individualizado de cuidados ${ }^{12,13}$.

Outra particularidade do cuidado das pessoas idosas com câncer é que essa população tende a valorizar a preservação da qualidade de vida (QV) e manutenção da independência mais do que os critérios de resposta dos ensaios clínicos tradicionais, como taxas de resposta geral, sobrevida livre de progressão ou aumento da expectativa de vida, ao tomar decisões da terapia oncológica ${ }^{14,15}$. No entanto, poucos estudos incorporam e avaliam a QV como desfecho de interesse do tratamento do câncer ${ }^{16}$.

Apesar dos benefícios da AGA e da recomendação das diretrizes internacionais ${ }^{3,7,12,17}$ para sua realização rotineira no cuidado das pessoas idosas com câncer, essas ferramentas requerem tempo e recursos consideráveis para serem integradas à prática, limitando seu uso generalizado, especialmente fora de ambientes acadêmicos especializados, sendo necessários dados mais robustos do seu benefício, a fim de reforçar essa abordagem ${ }^{13,18}$.

Desta forma, o objetivo desta revisão é avaliar a relação entre a AGA e a QV de pessoas idosas com câncer.

\section{MÉTODOS}

A metodologia adotada foi uma revisão integrativa da literatura, processo descrito por Whittemore e $\mathrm{Knafl}^{19}$, que permite a síntese de múltiplos estudos publicados e possibilita conclusões gerais a respeito de uma área particular de estudo ${ }^{20}$. 
O presente estudo foi realizado utilizando artigos publicados nas bases de dados científicos eletrônicos PubMed, MEDLINE, IBECS E LILACS, que abordassem as implicações da AGA na QV de pessoas idosas com câncer. A busca foi realizada no mês de março de 2020 por dois pesquisadores de forma independente com o objetivo de garantir a confiabilidade do presente estudo. Foram analisados estudos observacionais (transversais, caso-controle e coorte) e ensaios clínicos, no idioma inglês, espanhol ou português, publicados no período 01/05/2015 a 31/05/2020. Os estudos poderiam envolver pessoas idosas com qualquer tipo de câncer e submetidos a qualquer tipo de terapia oncológica. Como critério de exclusão, foram desconsiderados artigos que não contemplavam o tema, artigos de revisão, monografias, dissertações, teses, resumos em anais de eventos e capítulos de livro.

Foram utilizados os seguintes descritores: geriatric assessment, cancer, quality of life, evaluación geriátrica, cáncer, calidad de vida, avaliação geriátrica, câncer e qualidade de vida. Todos os descritores foram pesquisados através dos termos MeSH separadamente e, depois, foram cruzados com o operador booleano and. $\mathrm{O}$ cruzamento dos descritores geriatric assessment [Mesh] ANDcancer[Mesh] ANDquality of life no PubMed resultou em 263 registros; no MEDLINE em 229 registros; no IBECS em 1 registro; e no LILACS em 0 registros. Dos artigos localizados, 185 não contemplavam o tema proposto, 92 foram excluídos por serem artigos de revisão e 195 estavam duplicados nas plataformas de pesquisa (Figura 1).

Após a seleção dos artigos, foi elaborado um banco de dados que possibilitou organizar e compilar as seguintes informações dos estudos selecionados: título do artigo, ano de publicação, país de origem, desenho do estudo, objetivo, amostra, método e resultados (relação da AGA na QV de pessoas idosas com câncer).

As variáveis para análise dos resultados incluíram: instrumentos de AGA/QV e domínios alterados; associação entre comprometimento da AGA e QV; e mecanismo de correlação, seja por fornecer uma informação prognóstica ou por auxiliar na indicação de intervenção geriátrica específica. Posteriormente, foi realizado o agrupamento dos estudos por semelhança de conteúdo e os resultados foram interpretados com base na literatura correlata ao tema do estudo, possibilitando a síntese do conhecimento.

\section{RESULTADOS}

$\mathrm{Na}$ presente revisão integrativa foram analisados 21 artigos que atenderam aos critérios de seleção estabelecidos previamente. A seguir, o Quadro 1 apresenta um panorama geral dos artigos avaliados, considerando autoria, ano de publicação, país de origem, amostra, método, objetivo, instrumento e conclusões. 


\section{Revisão Integrativa da Literatura}

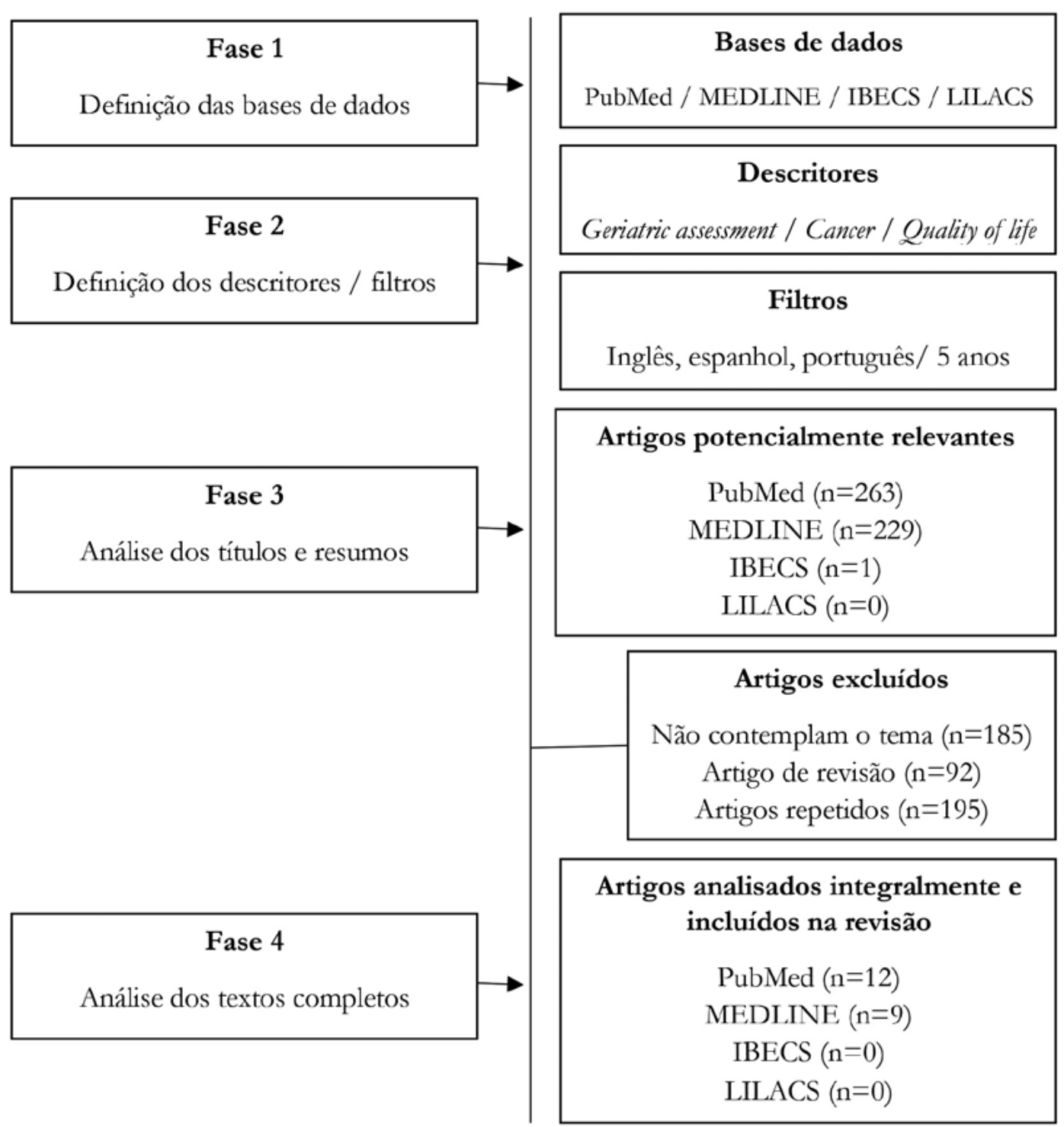

Figura 1. Fluxograma da revisão integrativa sobre evidências científicas entre avaliação geriátrica, câncer e qualidade de vida. 
Quadro 1. Apresentação da síntese de artigos incluídos na revisão integrativa.

\begin{tabular}{|c|c|c|c|c|c|}
\hline $\begin{array}{l}\text { Autores } \\
\text { Ano/País }\end{array}$ & Amostra & Método/Objetivo & Instrumento & Conclusões & $\begin{array}{l}\text { Relação da } \\
\text { AGA e QV }\end{array}$ \\
\hline $\begin{array}{l}\text { Pottel et al. }{ }^{21} \\
2015 \text { / Bélgica }\end{array}$ & $\begin{array}{l}\geq 65 \text { anos, } \\
\text { neoplasia de } \\
\text { cabeça e pescoço, } \\
\text { submetido } \\
\text { radioquimioterapia } \\
\text { / } \mathrm{N}=100\end{array}$ & $\begin{array}{l}\text { Coorte / } \\
\text { Determinar se AGA } \\
\text { é indicativa de QV } \\
\text { relacionada à saúde } \\
\text { em longo prazo e } \\
\text { sobrevida global }\end{array}$ & EQ-5D & $\begin{array}{l}\text { Pacientes } \\
\text { vulneráveis } \\
\text { apresentaram níveis } \\
\text { de QV relacionada } \\
\text { à saúde em longo } \\
\text { prazo inferiores }\end{array}$ & $\begin{array}{l}\text { A AGA foi um } \\
\text { preditor de QV }\end{array}$ \\
\hline $\begin{array}{l}\text { Baier et al. } .^{22} \\
2016 \text { / Alemanha }\end{array}$ & $\begin{array}{l}>70 \text { anos, qualquer } \\
\text { neoplasia, } \\
\text { submetido à } \\
\text { cirurgia com } \\
\text { intenção curativa / } \\
\mathrm{N}=200\end{array}$ & $\begin{array}{l}\text { Coorte / Avaliar o } \\
\text { impacto prognóstico } \\
\text { da AGA na } \\
\text { independência e QV } \\
\text { seis meses após a } \\
\text { cirurgia }\end{array}$ & $\begin{array}{l}\text { EORTC } \\
\text { QLQ-C30 }\end{array}$ & $\begin{array}{l}\text { A QV se } \\
\text { correlacionou com } \\
\text { índice de Karnofsky, } \\
\text { funcionamento } \\
\text { emocional e } \\
\text { atividades de vida } \\
\text { diária }\end{array}$ & $\begin{array}{l}\text { A AGA foi um } \\
\text { preditor de QV }\end{array}$ \\
\hline $\begin{array}{l}\text { Hempenius et al. } .^{23} \\
2016 \text { / Holanda }\end{array}$ & $\begin{array}{l}\geq 65 \text { anos, qualquer } \\
\text { neoplasia, } \\
\text { submetido à } \\
\text { cirurgia eletiva / } \\
\mathrm{N}=260\end{array}$ & $\begin{array}{l}\text { Ensaio clínico / } \\
\text { Avaliar os desfechos } \\
\text { em longo prazo de } \\
\text { uma intervenção } \\
\text { geriátrica para } \\
\text { prevenção de } \\
\text { delirium em idosos } \\
\text { frágeis }\end{array}$ & $\begin{array}{l}\text { Short Form-36 } \\
\text { score }\end{array}$ & $\begin{array}{l}\text { Não ocorreram } \\
\text { diferenças entre } \\
\text { o grupo de } \\
\text { intervenção e o } \\
\text { grupo de tratamento } \\
\text { usual para qualquer } \\
\text { um dos desfechos } \\
\text { três meses após a } \\
\text { alta }\end{array}$ & $\begin{array}{l}\text { A intervenção } \\
\text { geriátrica } \\
\text { baseada nos } \\
\text { achados da } \\
\text { AGA não } \\
\text { melhorou a } \\
\text { QV }\end{array}$ \\
\hline $\begin{array}{l}\text { Pergolotti et al. }{ }^{24} \\
2017 \text { / EUA }\end{array}$ & $\begin{array}{l}\geq 65 \text { anos, qualquer } \\
\text { neoplasia / } N=768\end{array}$ & $\begin{array}{l}\text { Coorte / Descrever } \\
\text { o status funcional } \\
\text { e QV e identificar } \\
\text { as associações } \\
\text { com variáveis } \\
\text { demográficas, } \\
\text { comorbidades e } \\
\text { status funcional }\end{array}$ & FACT-G & $\begin{array}{l}\text { A presença de } \\
\text { comorbidades e } \\
\text { níveis reduzidos } \\
\text { de atividade/ } \\
\text { capacidade funcional } \\
\text { se associaram com } \\
\text { piores níveis de QV }\end{array}$ & $\begin{array}{l}\text { A AGA foi um } \\
\text { preditor de QV }\end{array}$ \\
\hline $\begin{array}{l}\text { Ribi et al. }{ }^{25} \\
2017 \text { / Suíça }\end{array}$ & $\begin{array}{l}\text { Linfoma de células } \\
\text { B não elegíveis } \\
\text { para tratamento } \\
\text { intensivo / } \mathrm{N}=57\end{array}$ & $\begin{array}{l}\text { Ensaio clínico / } \\
\text { Caracterizar os } \\
\text { pacientes por } \\
\text { resposta objetiva } \\
\text { e sobrevida com } \\
\text { base na AGA e } \\
\text { QV e descrever as } \\
\text { alterações de QV } \\
\text { após o tratamento }\end{array}$ & $\begin{array}{l}\text { Domínios } \\
\text { avaliados: } \\
\text { bem-estar } \\
\text { físico, humor, } \\
\text { enfrentamento, } \\
\text { status } \\
\text { funcional, } \\
\text { cansaço, } \\
\text { náuseas / } \\
\text { vômitos e } \\
\text { distúrbios do } \\
\text { paladar }\end{array}$ & $\begin{array}{l}\text { Comprometimento } \\
\text { da AGA é um fator } \\
\text { importante dos } \\
\text { desfechos clínicos } \\
\text { e intervenções em } \\
\text { domínios geriátricos } \\
\text { específicos se } \\
\text { traduzem em } \\
\text { melhora na QV }\end{array}$ & $\begin{array}{l}\text { A AGA foi um } \\
\text { preditor de QV }\end{array}$ \\
\hline $\begin{array}{l}\text { Schmidt et al. }{ }^{26} \\
2017 \text { / Alemanha }\end{array}$ & $\begin{array}{l}\geq 70 \text { anos, qualquer } \\
\text { neoplasia / } \mathrm{N}=100\end{array}$ & $\begin{array}{l}\text { Ensaio clínico / } \\
\text { Avaliar o impacto } \\
\text { de um programa } \\
\text { de intervenção } \\
\text { baseado da AGA na } \\
\text { preservação da QV }\end{array}$ & $\begin{array}{l}\text { EORTC } \\
\text { QLQ-C30 }\end{array}$ & $\begin{array}{l}\text { A intervenção } \\
\text { geriátrica } \\
\text { demonstrou } \\
\text { benefício na } \\
\text { preservação da QV }\end{array}$ & $\begin{array}{l}\text { A intervenção } \\
\text { geriátrica } \\
\text { baseada nos } \\
\text { achados da } \\
\text { AGA auxilia } \\
\text { na preservação } \\
\text { da QV }\end{array}$ \\
\hline
\end{tabular}


Continuação do Quadro 1

\begin{tabular}{|c|c|c|c|c|c|}
\hline $\begin{array}{l}\text { Autores } \\
\text { Ano/País }\end{array}$ & Amostra & Método/Objetivo & Instrumento & Conclusões & $\begin{array}{l}\text { Relação da } \\
\text { AGA e QV }\end{array}$ \\
\hline $\begin{array}{l}\text { VanderWalde et } \\
\text { al. }^{27} \\
2017 \text { / EUA }\end{array}$ & $\begin{array}{l}\geq 65 \text { anos, } \\
\text { neoplasia de cabeça } \\
\text { e pescoço ou } \\
\text { pulmão realizando } \\
\text { radioterapia / } \\
\mathrm{N}=50\end{array}$ & $\begin{array}{l}\text { Coorte / Avaliar } \\
\text { a associação entre } \\
\text { o status funcional } \\
\text { com base na } \\
\text { AGA e resultados } \\
\text { de tolerância ao } \\
\text { tratamento }\end{array}$ & $\begin{array}{l}\text { EORTC } \\
\text { QLQ-C30 }\end{array}$ & $\begin{array}{l}\text { Não ocorreu } \\
\text { associação entre } \\
\text { disfunção e } \\
\text { tolerância, porém } \\
\text { a AGA alterada } \\
\text { associou-se com } \\
\text { declínio contínuo e } \\
\text { falta de recuperação } \\
\text { da QV }\end{array}$ & $\begin{array}{l}\text { A AGA foi um } \\
\text { preditor de QV }\end{array}$ \\
\hline $\begin{array}{l}\text { Goineau et al. } \\
2018 \text { / França }\end{array}$ & $\begin{array}{l}\geq 75 \text { anos, } \\
\text { neoplasia } \\
\text { de próstata, } \\
\text { submetidos à } \\
\text { radioterapia / } \\
\mathrm{N}=100\end{array}$ & $\begin{array}{l}\text { Coorte / Avaliar } \\
\text { o efeito da } \\
\text { radioterapia na QV e } \\
\text { identificar preditores } \\
\text { de redução da QV }\end{array}$ & $\begin{array}{l}\text { EORTC } \\
\text { QLQ-C30 }\end{array}$ & $\begin{array}{l}\text { A radioterapia para } \\
\text { câncer de próstata } \\
\text { foi bem tolerada } \\
\text { nessa população e } \\
\text { não foi encontrado } \\
\text { fator preditivo } \\
\text { para determinar } \\
\text { quais pacientes } \\
\text { apresentariam QV } \\
\text { prejudicada após a } \\
\text { radioterapia }\end{array}$ & $\begin{array}{l}\text { A AGA não foi } \\
\text { um preditor de } \\
\text { QV }\end{array}$ \\
\hline $\begin{array}{l}\text { Phaibulvatanapong } \\
\text { et al. }^{29} \\
2018 \text { / Tailândia }\end{array}$ & $\begin{array}{l}\geq 70 \text { anos, qualquer } \\
\text { neoplasia em } \\
\text { tratamento } \\
\text { sistêmico / } \mathrm{N}=151\end{array}$ & $\begin{array}{l}\text { Coorte / Avaliar } \\
\text { os fatores que } \\
\text { predispõem } \\
\text { toxicidade } \\
\text { relacionada à } \\
\text { quimioterapia e QV }\end{array}$ & FACT-G & $\begin{array}{l}\text { O performace status } \\
\text { e a presença de } \\
\text { comorbidade } \\
\text { associaram-se com } \\
\text { maior incidência de } \\
\text { eventos adversos } \\
\text { graves e pior QV }\end{array}$ & $\begin{array}{l}\text { A AGA foi um } \\
\text { preditor de QV }\end{array}$ \\
\hline $\begin{array}{l}\text { Puts et al. }{ }^{30} \\
2018 \text { / Canadá }\end{array}$ & $\begin{array}{l}\geq 70 \text { anos, } \\
\text { neoplasia } \\
\text { gastrointestinal, } \\
\text { geniturinário ou de } \\
\text { mama EC II-IV, } \\
\text { antes do início de } \\
\text { quimioterapia / } \\
\mathrm{N}=61\end{array}$ & $\begin{array}{l}\text { Ensaio clínico } \\
\text { / Explorar a } \\
\text { viabilidade e o } \\
\text { impacto da AGA } \\
\text { e de um plano de } \\
\text { cuidados integrados } \\
\text { na QV e nas decisões } \\
\text { de tratamento } \\
\text { oncológico }\end{array}$ & $\begin{array}{l}\text { EORTC } \\
\text { QLQ-C30 }\end{array}$ & $\begin{array}{l}\text { Pacientes que } \\
\text { receberam suporte } \\
\text { baseado na AGA } \\
\text { tiveram melhores } \\
\text { níveis de QV }\end{array}$ & $\begin{array}{l}\text { A intervenção } \\
\text { geriátrica } \\
\text { baseada nos } \\
\text { achados da } \\
\text { AGA auxilia } \\
\text { na preservação } \\
\text { da QV }\end{array}$ \\
\hline $\begin{array}{l}\text { Jeppesen et al. }{ }^{31} \\
2018 \text { / Dinamarca }\end{array}$ & $\begin{array}{l}\text { Neoplasia de } \\
\text { pulmão T1- } \\
\text { 2N0M0 não } \\
\text { candidatos a } \\
\text { tratamento } \\
\text { cirúrgico / N=51 }\end{array}$ & $\begin{array}{l}\text { Ensaio clínico / } \\
\text { Investigar se AGA } \\
\text { como parte de } \\
\text { uma ferramenta } \\
\text { intervencionista } \\
\text { pode impactar na } \\
\text { QV e sobrevida } \\
\text { global }\end{array}$ & EQ-5D & $\begin{array}{l}\text { A AGA não } \\
\text { impactou na QV } \\
\text { e sobrevida global } \\
\text { nessa população }\end{array}$ & $\begin{array}{l}\text { A intervenção } \\
\text { geriátrica } \\
\text { baseada nos } \\
\text { achados da } \\
\text { AGA não } \\
\text { melhorou a } \\
\text { QV }\end{array}$ \\
\hline $\begin{array}{l}\text { Kirkhus et al. }{ }^{14} \\
2019 \text { / Noruega }\end{array}$ & $\begin{array}{l}\geq 70 \text { anos, qualquer } \\
\text { neoplasia em } \\
\text { tratamento } \\
\text { sistêmico / } \mathrm{N}=288\end{array}$ & $\begin{array}{l}\text { Coorte / } \\
\text { Identificar fatores } \\
\text { potencialmente } \\
\text { modificáveis que } \\
\text { afetam a função física } \\
\text { e a QV durante o } \\
\text { tratamento do câncer }\end{array}$ & $\begin{array}{l}\text { EORTC } \\
\text { QLQ-C30 }\end{array}$ & $\begin{array}{l}\text { Sintomas } \\
\text { depressivos, } \\
\text { mobilidade reduzida } \\
\text { e sintomas físicos } \\
\text { aumentaram o risco } \\
\text { de decréscimos nos } \\
\text { escores de QV }\end{array}$ & $\begin{array}{l}\text { A AGA foi um } \\
\text { preditor de QV }\end{array}$ \\
\hline
\end{tabular}


Continuação do Quadro 1

\begin{tabular}{|c|c|c|c|c|c|}
\hline $\begin{array}{l}\text { Autores } \\
\text { Ano/País }\end{array}$ & Amostra & Método/Objetivo & Instrumento & Conclusões & $\begin{array}{l}\text { Relação da } \\
\text { AGA e QV }\end{array}$ \\
\hline $\begin{array}{l}\text { Kirkhus et al. }{ }^{32} \\
2019 \text { / Noruega }\end{array}$ & $\begin{array}{l}\geq 70 \text { anos, qualquer } \\
\text { neoplasia / } \mathrm{N}=288\end{array}$ & $\begin{array}{l}\text { Coorte / Investigar } \\
\text { se a fragilidade } \\
\text { identificada por uma } \\
\text { AGA foi associado } \\
\text { a um maior risco } \\
\text { de deterioração } \\
\text { da QV durante } \\
\text { o tratamento e } \\
\text { acompanhamento } \\
\text { do câncer }\end{array}$ & $\begin{array}{l}\text { EORTC } \\
\text { QLQ-C30 }\end{array}$ & $\begin{array}{l}\text { Pacientes } \\
\text { frágeis tiveram } \\
\text { significativamente } \\
\text { pior funcionamento } \\
\text { físico e QV durante } \\
\text { o acompanhamento }\end{array}$ & $\begin{array}{l}\text { A AGA foi um } \\
\text { preditor de QV }\end{array}$ \\
\hline $\begin{array}{l}\text { Mohile et al. }{ }^{3} \\
2019 \text { / EUA }\end{array}$ & $\begin{array}{l}\geq 70 \text { anos, qualquer } \\
\text { neoplasia, algum } \\
\text { domínio da AGA } \\
\text { comprometido / } \\
\mathrm{N}=541\end{array}$ & $\begin{array}{l}\text { Ensaio clínico } \\
\text { / Determinar } \\
\text { se fornecer } \\
\text { recomendações } \\
\text { guiadas pela AGA } \\
\text { para oncologistas } \\
\text { pode melhorar a } \\
\text { comunicação sobre } \\
\text { as preocupações } \\
\text { relacionadas ao } \\
\text { envelhecimento }\end{array}$ & FACT-G & $\begin{array}{l}\text { Avaliação } \\
\text { geriátrica melhora } \\
\text { a comunicação } \\
\text { centrada no paciente } \\
\text { sobre preocupações } \\
\text { relacionadas ao } \\
\text { envelhecimento, } \\
\text { porém não } \\
\text { modificou a QV }\end{array}$ & $\begin{array}{l}\text { A intervenção } \\
\text { geriátrica } \\
\text { baseada nos } \\
\text { achados da } \\
\text { AGA não } \\
\text { melhorou a } \\
\text { QV }\end{array}$ \\
\hline $\begin{array}{l}\text { Quinten et al. }{ }^{33} \\
2019 \text { / Bélgica }\end{array}$ & $\begin{array}{l}\geq 70 \text { anos, qualquer } \\
\text { neoplasia, } \\
\text { submetidos à } \\
\text { quimioterapia ou } \\
\text { cirurgia, G8 } \leq 14 \text { / } \\
\mathrm{N}=1424\end{array}$ & $\begin{array}{l}\text { Coorte / } \\
\text { Determinar as } \\
\text { estimativas mínimas } \\
\text { de diferenças } \\
\text { clinicamente } \\
\text { importantes de } \\
\text { QV e avaliar } \\
\text { características } \\
\text { prognósticas para } \\
\text { estas mudanças na } \\
\text { QV }\end{array}$ & $\begin{array}{l}\text { EORTC } \\
\text { QLQ-C30 }\end{array}$ & $\begin{array}{l}\text { As estimativas } \\
\text { mínimas de } \\
\text { diferenças } \\
\text { clinicamente } \\
\text { importantes da QV } \\
\text { variam conforme } \\
\text { o instrumento } \\
\text { e o tratamento, } \\
\text { mas podem } \\
\text { ser usadas para } \\
\text { avaliar mudanças } \\
\text { significativas na QV }\end{array}$ & $\begin{array}{l}\text { A AGA foi um } \\
\text { preditor de QV }\end{array}$ \\
\hline $\begin{array}{l}\text { Williams et al. } \\
2019 \text { / EUA }\end{array}$ & $\begin{array}{l}\geq 65 \text { anos, } \\
\text { neoplasia de mama } \\
/ \mathrm{N}=190\end{array}$ & $\begin{array}{l}\text { Coorte / Avaliar } \\
\text { a associação entre } \\
\text { fragilidade e QV }\end{array}$ & PROMIS® & $\begin{array}{l}\text { Fragilidade em } \\
\text { mulheres idosas } \\
\text { com câncer de } \\
\text { mama foi associada } \\
\text { a resultados piores } \\
\text { de QV }\end{array}$ & $\begin{array}{l}\text { A AGA foi um } \\
\text { preditor de QV }\end{array}$ \\
\hline $\begin{array}{l}\text { de Boer et al. }{ }^{6} \\
2020 / \text { Holanda }\end{array}$ & $\begin{array}{l}\geq 70 \text { anos, } \\
\text { neoplasia de mama } \\
\text { metastática / } \\
\mathrm{N}=100\end{array}$ & $\begin{array}{l}\text { Coorte / Avaliar } \\
\text { a prevalência } \\
\text { de doenças } \\
\text { psicossociais } \\
\text { e as mudanças } \\
\text { longitudinais no } \\
\text { status funcional, } \\
\text { funcionamento } \\
\text { psicossocial e QV }\end{array}$ & $\begin{array}{l}\text { EORTC } \\
\text { QLQ-C30 }\end{array}$ & $\begin{array}{l}\text { Elevada prevalência } \\
\text { de transtornos } \\
\text { psiquiátricos nesta } \\
\text { população; sua } \\
\text { identificação, através } \\
\text { da AGA, pode } \\
\text { melhorar a QV }\end{array}$ & $\begin{array}{l}\text { A intervenção } \\
\text { geriátrica } \\
\text { baseada nos } \\
\text { achados da } \\
\text { AGA auxilia } \\
\text { na preservação } \\
\text { da QV }\end{array}$ \\
\hline
\end{tabular}

continua 
Continuação do Quadro 1

\begin{tabular}{|c|c|c|c|c|c|}
\hline $\begin{array}{l}\text { Autores } \\
\text { Ano/País }\end{array}$ & Amostra & Método/Objetivo & Instrumento & Conclusões & $\begin{array}{l}\text { Relação da } \\
\text { AGA e QV }\end{array}$ \\
\hline $\begin{array}{l}\text { Mian et al. }{ }^{16} \\
2020 \text { / Canadá }\end{array}$ & $\begin{array}{l}\geq 65 \text { anos, } \\
\text { diagnóstico } \\
\text { recente de mieloma } \\
\text { múltiplo / N=40 }\end{array}$ & $\begin{array}{l}\text { Coorte / } \\
\text { Compreender as } \\
\text { mudanças ocorridas } \\
\text { nos domínios } \\
\text { geriátricos e nos } \\
\text { parâmetros de } \\
\text { QV ao longo } \\
\text { do tratamento } \\
\text { oncológico }\end{array}$ & FACT-G & $\begin{array}{l}\text { Nesta população, } \\
\text { a QV permaneceu } \\
\text { estável no período } \\
\text { de } 6 \text { meses de } \\
\text { acompanhamento; } \\
\text { o teste Timed Up and } \\
\text { Go pode fornecer } \\
\text { um indicador } \\
\text { dinâmico do status } \\
\text { funcional e QV }\end{array}$ & $\begin{array}{l}\text { A AGA foi um } \\
\text { preditor de QV }\end{array}$ \\
\hline $\begin{array}{l}\text { Nipp et al. }{ }^{13} \\
2020 / \text { EUA }\end{array}$ & $\begin{array}{l}\geq 65 \text { anos, } \\
\text { neoplasia } \\
\text { gastrointestinal } \\
\text { ou pulmonar } \\
\text { incurável / } \mathrm{N}=62\end{array}$ & $\begin{array}{l}\text { Ensaio clínico } \\
\text { / Determinar a } \\
\text { viabilidade de } \\
\text { uma intervenção } \\
\text { transdisciplinar } \\
\text { baseada em uma } \\
\text { AGA }\end{array}$ & FACT-G & $\begin{array}{l}\text { A intervenção } \\
\text { transdisciplinar } \\
\text { direcionada às } \\
\text { necessidades de } \\
\text { cuidados de pessoas } \\
\text { idosas mostraram } \\
\text { estimativas } \\
\text { encorajadoras para } \\
\text { melhorar a QV }\end{array}$ & $\begin{array}{l}\text { A intervenção } \\
\text { geriátrica } \\
\text { baseada nos } \\
\text { achados da } \\
\text { AGA auxilia } \\
\text { na preservação } \\
\text { da QV }\end{array}$ \\
\hline $\begin{array}{l}\text { Nipp et al. }{ }^{8} \\
2020 / \text { EUA }\end{array}$ & $\begin{array}{l}\geq 70 \text { anos, } \\
\text { diagnóstico recente } \\
\text { de neoplasia } \\
\text { gastrointestinal } \\
\text { incurável } \\
\mathrm{N}=132\end{array}$ & $\begin{array}{l}\text { Coorte / } \\
\text { Determinar se } \\
\text { categorizar os } \\
\text { pacientes como } \\
\text { vulneráveis por } \\
\text { uma AGA poderia } \\
\text { identificar aqueles } \\
\text { com piores } \\
\text { resultados de saúde }\end{array}$ & $\begin{array}{l}\text { EORTC } \\
\text { QLQ-C30 }\end{array}$ & $\begin{array}{l}\text { Pacientes } \\
\text { identificados como } \\
\text { vulneráveis pela } \\
\text { AGA apresentam } \\
\text { pior QV e sobrevida } \\
\text { global }\end{array}$ & $\begin{array}{l}\text { A AGA foi um } \\
\text { preditor de QV }\end{array}$ \\
\hline $\begin{array}{l}\text { Quinten et al. }{ }^{15} \\
2020 / \text { Bélgica }\end{array}$ & $\begin{array}{l}\geq 70 \text { anos, } \\
\text { neoplasia de mama } \\
\text { em estádio inicial } \\
\mathrm{N}=109\end{array}$ & $\begin{array}{l}\text { Caso-controle / } \\
\text { Avaliar a relação } \\
\text { entre AGA e QV }\end{array}$ & $\begin{array}{l}\text { EORTC } \\
\text { QLQ-C30 }\end{array}$ & $\begin{array}{l}\text { Medidas funcionais } \\
\text { da AGA são } \\
\text { fortemente } \\
\text { correlacionadas } \\
\text { com funcionamento } \\
\text { autorrelatado do } \\
\text { paciente; a AGA } \\
\text { alterada inicial tem } \\
\text { uma probabilidade } \\
\text { modesta de prever a } \\
\text { deterioração da QV }\end{array}$ & $\begin{array}{l}\text { A AGA foi um } \\
\text { preditor de QV }\end{array}$ \\
\hline
\end{tabular}

AGA: avaliação geriátrica ampla; QV: qualidade de vida; Euro Quality of Life Instrument-5D: EQ-5D; European Organisation for Research and Treatment of Cancer Quality of Life Core Questionnaire-C30: EORTC-QLQ-C30; Functional Assessment of Cancer Therapy-General: FACT-G; Patient-Reported Outcomes Measurement Information System®: PROMIS®; Estados Unidos da América: EUA. 


\section{DISCUSSÃO}

Distúrbios das funções físicas, deficit nutricionais e problemas psicossociais ocorrem em cerca de $20-40 \%$ das pessoas idosas com diagnóstico de câncer $^{34-37}$. Tais alterações, rastreadas através da aplicação da AGA, são capazes de identificar pacientes frágeis, nos quais as manifestações relacionadas à doença neoplásica e ao tratamento oncológico estão associadas a uma carga substancial de sintomas e podem reduzir o estado funcional e ameaçar a capacidade de viver de forma independente das pessoas idosas, comprometendo de forma negativa a QV durante o curso da doença ${ }^{32}$.

Em consonância, diversos trabalhos avaliados demonstraram que os pacientes com comprometimento em algum domínio da AGA apresentaram pior QV em comparação com pacientes sem comprometimento, ou seja, apresentavam maior deterioração nos índices de QV durante o seguimento $5,6,8,15,21,22,24,25$. Pottel et al. ${ }^{21}$ identificaram que a classificação de vulnerabilidade, a partir do comprometimento em dois ou mais domínios da AGA, foi um preditor independente para pontuações inferiores nos índices de QV em uma população de 100 pessoas idosas com neoplasia de cabeça e pescoço, em um período de seguimento de até 36 meses.

Considerando que AGA é um processo de abordagem multidimensional, diferentes domínios podem estar alterados e predizer essa maior susceptibilidade ao comprometimento da QV das pessoas idosas com câncer. Enquanto alguns trabalhos utilizaram a classificação de pacientes como vulneráveis ou frágeis, através de uma graduação específica $^{5,8,21,25,32}$, outros avaliaram o impacto dos diferentes domínios individualmente, sendo que os fatores geriátricos mais frequentemente preditivos para uma diminuição relevante na QV foram a capacidade funcional (índice de Karnofsky, atividades da vida diária ou ECOG performance status), o funcionamento emocional e o índice de massa corporal $1^{6,14,15,22,24,29}$. Apenas um trabalho ${ }^{21}$ demonstrou que quanto maior o número de domínios alterados da AGA, maior era o impacto na QV.

A maioria dos trabalhos avaliados descreveu o escore de QV global. Nos trabalhos que relatam os domínios aferidos separadamente, o comprometimento da QV de pessoas idosas com disfunção da AGA frequentemente ocorre nas diversas esferas, incluindo função física, emocional, cognitiva e social, desempenho de papéis e sintomas ${ }^{5,8,14,15,27,32}$.

Apesar dessa associação lógica entre vulnerabilidade e piora da QV de pessoas idosas com câncer, alguns trabalhos avaliados não demonstraram uma associação estatisticamente significativa entre alterações na AGA e o comprometimento da QV. Goineau et al. ${ }^{28}$ aplicaram a AGA em uma coorte de pessoas idosas que foram submetidos à radioterapia modulada por intensidade para câncer de próstata. Nenhum parâmetro geriátrico foi preditivo de QV prejudicada após o tratamento, porém nesse estudo a radioterapia foi bem tolerada e a QV foi preservada na maioria dos pacientes. Da mesma forma, estudo conduzido por Mohile et al. ${ }^{18}$ não detectou diferenças estatisticamente significativas entre os grupos na pontuação da escala de QV, independentemente dos valores basais da AGA. Apesar disso, o estudo demonstrou que incluir a AGA nas consultas de oncologia melhorava a comunicação centrada no paciente sobre preocupações relacionadas com o envelhecimento e a satisfação do paciente e do cuidador.

Ainda, Kirkhus et al. ${ }^{32}$ acompanharam uma coorte de pessoas idosas com câncer e verificaram que embora a maioria dos aspectos de QV fossem piores em pacientes classificados como frágeis pela AGA, as mudanças seguiam um curso semelhante aos pacientes não frágeis, porém, como os primeiros apresentavam valores basais de QV inferiores, mudanças na mesma magnitude afetavam esses pacientes mais profundamente.

O impacto do processo do envelhecimento nos mecanismos de farmacodinâmica e farmacocinética dos medicamentos é amplamente conhecido, resultando assim na minimização da tolerância do tecido normal aos agentes antineoplásico e maior toxicidade, o que desempenha um papel importante na QV dessa população ${ }^{29,38}$. Desta forma, a capacidade da AGA de prever a tolerabilidade ao tratamento oncológico é de crucial importância, pois pode auxiliar na antecipação de medidas que visem prevenir a toxicidade ao tratamento. Phaibulvatanapong et al. ${ }^{29}$ 
avaliaram que distúrbios da capacidade funcional, do estado nutricional e a presença de comorbidades foram fatores considerados preditivos de toxicidade grave e QV prejudicada em pessoas idosas com câncer.

Além do impacto na tolerância ao tratamento oncológico, essa diminuição progressiva na reserva funcional de múltiplos sistemas orgânicos, associada ao envelhecimento, influencia também a capacidade de recuperação do indivíduo às toxicidades agudas, resultando em deficit funcionais prolongados e, consequentemente, na diminuição da $\mathrm{QV}^{24,29,30}$. Neste contexto, a AGA pode representar também um preditor da incapacidade de recuperação da QV após terapia antineoplásica. De fato, dois estudos ${ }^{21,27}$ demonstraram que pessoas idosas com disfunção basal na AGA, além de apresentarem uma queda mais importante nos índices de QV, eram mais propensos a manter os níveis de QV reduzidos mesmo após o término do tratamento oncológico.

Assim, o possível benefício do tratamento oncológico em pacientes idosos deve ser pesado aos seus potenciais danos e, como as opções de tratamento do câncer em pessoas idosas são baseadas em extrapolações de evidências derivadas de ensaios clínicos que envolvem predominantemente pacientes mais jovens ou idosos sem comprometimento funcional, a AGA pode representar uma ferramenta útil na decisão da terapia ${ }^{18,29,39}$. Estudos prévios descrevem que os achados da AGA podem levar a modificações no tratamento oncológico em aproximadamente $30 \%$ dos planos de terapia de pessoas idosas, visando garantir uma melhor tolerância e, consequentemente, um impacto positivo na $\mathrm{QV}^{40,41}$. Um trabalho avaliado demonstrou que a AGA é capaz de auxiliar nesse processo de individualização do tratamento oncológico ocasionando um impacto positivo da $\mathrm{QV}^{33}$.

Avaliações sistemáticas de sintomas, intervenções direcionadas às preocupações geriátricas específicas e cuidados interdisciplinares de apoio podem melhorar os desfechos de pessoas idosas com câncer. Por isso, recomenda-se que a AGA seja seguida por um plano de cuidados integrados para abordar as questões identificadas ${ }^{30,32}$. Estudo de Schimidt et al. ${ }^{26}$ realizou um teste-piloto de intervenção com terapia de suporte intensificada durante os cuidados de pessoas idosas com câncer avançado e os resultados demonstraram que a medida global de QV da maioria dos participantes (72\%) melhorou ou permaneceu estável. Outros trabalhos selecionados que avaliaram a implantação de intervenções direcionadas conforme os achados da AGA também evidenciaram melhores desfechos de QV nos pacientes designados para o grupo de intervenção do que aqueles designados para os cuidados habituais ${ }^{13,30}$.

Jeppensen et al. ${ }^{31}$ utilizaram a AGA como parte de uma ferramenta intervencionista para otimizar o estado geral de saúde dos pacientes incluídos e, apesar de não demonstrarem diferenças estatisticamente significativas entre os grupos, ocorreu uma redução nas pontuações de QV a longo prazo no grupo que não recebeu a intervenção geriátrica, a qual não ocorreu no grupo da intervenção. Apenas um estudo $^{23}$, que realizou uma intervenção geriátrica direcionada aos fatores de risco para delirium pós-operatório em pacientes idosos classificados como frágeis submetidos à cirurgia para um tumor sólido, não demonstrou benefício no desfecho de QV no seguimento desses pacientes, porém a taxa de incidência de delirium, que foi abaixo do esperado, e o alto padrão de cuidados básicos do grupo controle podem ter influenciado os resultados em longo prazo.

A utilidade da AGA em melhorar os resultados das pessoas idosas com câncer foi descrita em estudos de revisão prévios ${ }^{42-44}$, demonstrando seu benefício em diferentes desfechos, como melhora da tolerância ao tratamento e sobrevida global. No entanto, dados sobre as implicações da abordagem na QV dessa população são escassos, demonstrando a importância desse trabalho. O estudo teve como limitação, porém, o fato de não ter utilizado todas as bases de dados da área da saúde, englobado uma amostra menor de trabalhos. No entanto, a revisão permitiu demonstrar lacunas na literatura, particularmente a carência de estudos com delineamentos mais objetivos para a compreensão dos benefícios e da viabilidade da AGA e que avaliem especificamente o seu impacto na QV, desfecho de relevância para a população idosa, a fim de fornecer informações relevantes que possam ser usadas para facilitar as decisões de tratamento. Durante a busca nas plataformas de pesquisa, foram identificados três ensaios clínicos que estão em 
andamento e apresentam um melhor delineamento e maior amostra para oferecer dados mais robustos a essa temática (NCT02704832; NCT02284308; NCT02748811) ${ }^{45-47}$.

\section{CONCLUSÃO}

A partir da análise da produção científica sobre a relação da AGA e QV em pessoas idosas com câncer, evidenciou-se a importância da avaliação integral dessas pessoas, tanto para definições prognósticas e de tolerabilidade ao tratamento, quanto para auxiliar

\section{REFERÊNCIAS}

1. Bray F, Ferlay J, Soerjomataram I, Siegel RL, Torre LA, Jemal A. Global cancer statistics 2018: GLOBOCAN estimates of incidence and mortality worldwide for 36 cancers in 185 countries. CA Cancer J Clin. 2018;68(6):394-424.

2. Zhang X, Meng X, Chen Y, Leng SX, Zhang H. The biology of aging and cancer: frailty, inflammation, and immunity. Cancer J. 2017;23(4):201-5.

3. Mohile SG, Dale W, Somerfield MR, Schonberg MA, Boyd CM, Burhenn PS, et al. Practical assessment and management of vulnerabilities in older patients receiving chemotherapy: ASCO Guideline for Geriatric Oncology. J Clin Oncol. 2018;36(22):2326-47.

4. Miller KD, Nogueira L, Mariotto AB, Rowland JH, Yabroff KR, Alfano CM, et al. Cancer treatment and survivorship statistics, 2019. CA Cancer J Clin. 2019;69(5):363-85.

5. Williams GR, Deal AM, Sanoff HK, Nyrop KA, Guerard EJ, Pergolotti M, et al. Frailty and healthrelated quality of life in older women with breast cancer. Support Care Cancer. 2019;27(7):2693-98.

6. de Boer AZ, Derks MGM, de Glas NA, Bastiaannet E, Liefers GJ, Stiggelbout AM. Metastatic breast cancer in older patients: A longitudinal assessment of geriatric outcomes. J Geriatr Oncol. 2020;11(6):969-75.

7. Sarrió RG, Rebollo MA, Garrido MJM, GuillénPonce G, Blanco R, Flores EG, et al. General recommendations paper on the management of older patients with cancer: the SEOM geriatric oncology task force's position statement. Clin Transl Oncol. 2018;20(10):1246-51. na decisão do tratamento oncológico e orientação de intervenções de suporte. Através dessas diferentes aplicações, observou-se que a AGA auxilia na preservação da QV dessa população.

Estes resultados sugerem a importância de desenvolver estratégias para incorporação da AGA no cuidado das pessoas idosas com câncer, a fim de garantir uma abordagem abrangente desses indivíduos e o melhor cuidado dessa população vulnerável, priorizando a melhoria da QV.

Editado por: Maria Luiza Diniz de Sousa Lopes
8. Nipp RD, Thompson LL, Temel B, Fuh CX, Server C, Kay PS, et al. Screening tool identifies older adults with cancer at risk for poor outcomes. J Natl Compr Canc Netw. 2020;18(3):305-13.

9. Hamaker ME, Seynaeve C, Wymenga ANM, van Tinteren H, Nortier JHR, Maartense E, et al. Baseline comprehensive geriatric assessment is associated with toxicity and survival in elderly metastatic breast cancer patients receiving single-agent chemotherapy: results from the OMEGA study of the Dutch breast cancer trialists' group. Breast .2014;23(1):81-7.

10. Aaldriks AA, Maartense E, Nortier HJWR, van der Geest LGM, le Cessie S, Tanis BC, et al. Prognostic factors for the feasibility of chemotherapy and the Geriatric Prognostic Index (GPI) as risk profile for mortality before chemotherapy in the elderly. Acta Oncol. 2016;55(1):15-23.

11. Denewet N, de Breucker S, Luce S, Kennes B, Higuet $\mathrm{S}$, Pepersack T. Comprehensive geriatric assessment and comorbidities predict survival in geriatric oncology. Acta Clin Belg. 2016;71(4):206-13.

12. Wildiers H, Heeren P, Puts M, Topinkova E, JanssenHeijnen MLG, Extermann M, et al. International Society of Geriatric Oncology consensus on geriatric assessment in older patients with cancer. J Clin Oncol. 2014;32(24):2595-603.

13. Nipp RD, Temel B, Fuh CX, Kay P, Landay S, Lage $\mathrm{D}$, et al. Pilot randomized trial of a transdisciplinary geriatric and palliative care intervention for older adults with cancer. J Natl Compr Canc Netw. 2020;18(5):591-8. 
14. Kirkhus L, Harneshaug M, Benth LS, Gronberg $\mathrm{BH}$, Rostoft S, Bergh S, et al. Modifiable factors affecting older patients' quality of life and physical function during cancer treatment. J Geriatr Oncol. 2019;10(6):904-12.

15. Quinten C, Kenis C, Hamaker M, Coolbrandt A, Brouwers B, Dal Lago L, el al. The added value of geriatric assessment in evaluating a patient's HealthRelated Quality-of-Life: a study in $\geq 70$-year-old earlystage invasive breast cancer patients. Eur J Cancer Care (Engl). 2020;e13278.

16. Mian H, Pond GR, Tuchman SA, Fiala MA, Wildes TM. Geriatric assessment and quality of life changes in older adults with newly diagnosed multiple myeloma undergoing treatment. J Geriatr Oncol. 2020;11(8):1279-84.

17. Hurria A, Levit LA, Dale W, Mohile SG, Muss HB, Fehrenbacher L, et al. Improving the evidence base for treating older adults with cancer: American Society of Clinical Oncology Statement. J Clin Oncol. 2015;33(32):3826-33.

18. Mohile SG, Epstein RM, Hurria A, Heckler CE, Canin B, Culakova E, et al. Communication with older patients with cancer using geriatric assessment: a Cluster-Randomized Clinical Trial From the National Cancer Institute Community Oncology Research Program. JAMA Oncol. 2019;6(2):1-9.

19. Whittemore R, Knafl K. The integrative review: Updated methodology. J Adv Nurs. 2005;52(5):546-53.

20. Hopia H, Latvala E, Liimatainen L. Reviewing the methodology of an integrative review. Scand J Caring Sci. 2016;30(4):662-9.

21. Pottel L, Lycke M, Boterberg T, Pottel H, Goethals L, Duprez F, et al. G-8 indicates overall and qualityadjusted survival in older head and neck cancer patients treated with curative radiochemotherapy. BMC Cancer. 2015;15:875.

22. Baier P, Ihorst G, Wolff-Vorbeck G, Hüll M, Hopt U, Deschler B. Independence and health related quality of life in 200 onco-geriatric surgical patients within 6 months of follow-up: Who is at risk to lose? Eur J Surg Oncol. 2016;42(12):1890-7.

23. Hempenius L, Slaets JPJ, van Asselt D, de Bock TH, Wiggers T, van Leeuwen BL. Long Term Outcomes of a Geriatric Liaison Intervention in Frail Elderly Cancer Patients. PLoS ONE. 2016;11(2):e0143364.

24. Pergolotti M, Deal AM, Williams GR, Bryant AL, Bensen JT, Muss HB, et al. Activities, function, and health-related quality of life (HRQOL) of older adults with cancer. J Geriatr Oncol. 2017;8(4):249-54.
25. Ribi K, Rondeau S, Hitz F, Mey U, Enoiu M, Pabst T, et al. Cancer-specific geriatric assessment and quality of life: important factors in caring for older patients with aggressive B-cell lymphoma. Support Care Cancer. 2017;25(9):2833-42.

26. Schmidt H, Boese S, Lampe K, Jordan K, Fiedler E, Müller-Werdan U, et al. Trans sectoral care of geriatric cancer patients based on comprehensive geriatric assessment and patient-reported quality of life - Results of a multicenter study to develop and pilot test a patient-centered interdisciplinary care concept for geriatric oncology patients (PIVOG). J Geriatr Onco.1 2017;8(4):262-70.

27. VanderWalde NA, Deal AM, Comitz E, Stravers L, Muss H, Reeve BB, et al. Geriatric assessment as a predictor of tolerance, quality of life, and outcomes in older patients with head and Neck Cancers and Lung Cancers receiving radiation therapy. Int J Radiat Oncol Biol Phys. 2017;98(4):850-7.

28. Goineau A, Campion L, d'Aillières B, Vié B, Ghesquière A, Béra G, et al. Comprehensive Geriatric Assessment and quality of life after localized prostate cancer radiotherapy in elderly patients. PLoS ONE. 2018;13(4):e0194173.

29. Phaibulvatanapong E, Srinonprasert V, Ithimakin S. Risk factors for chemotherapy-related toxicity and adverse events in elderly thai cancer patients: a prospective study. Oncology. 2018;94(3):149-60.

30. Puts MTE, Sattar S, Kulik M, MacDonald ME, McWatters K, Lee K, et al. A randomized phase II trial of geriatric assessment and management for older cancer patients. Support Care Cancer. 2018;26(1):109-17.

31. Jeppesen SS, Matzen LE, Brink C, Bliucukiene R, Kasch S, Schytte T, et al. Impact of comprehensive geriatric assessment on quality of life, overall survival, and unplanned admission in patients with nonsmall cell lung cancer treated with stereotactic body radiotherapy. J Geriatr Oncol. 2018;9(6):575-82.

32. Kirkhus L, Benth JS, Gronberg BH, Hjermstad MJ, Rostoft S, Harneshaug M, et al. Frailty identified by geriatric assessment is associated with poor functioning, high symptom burden and increased risk of physical decline in older cancer patients: Prospective observational study. Palliat Med. 2019;33(3):312-22.

33. Quinten C, Kenis C, Decoster L, Debruyne PR, de Groof I, Focan C, et al. Determining clinically important differences in health-related quality of life in older patients with cancer undergoing chemotherapy or surgery. Qual Life Res. 2019;28(3):663-76. 
34. Kenis C, Decoster L, Bastin J, Bode H, Van Puyvelde $\mathrm{K}$, de Greve J, et al. Functional decline in older patients with cancer receiving chemotherapy: a multi- center prospective study. J Geriatr Oncol. 2017;8(3):196-205.

35. Decoster L, Kenis C, Schallier D, Vansteenkiste J, Nackaerts K, Vanacker L, et al. Geriatric assessment and functional decline in older patients with lung cancer. Lung. 2017;195(5):619-26.

36. Hoppe S, Rainfray M, Fonck M, Hoppenreys L, Blanc JF, Ceccaldi J, et al. Functional decline in older patients with cancer receiving first-line chemotherapy. J Clin Oncol. 2013;31(31):3877-82.

37. van Abbema D, van Vuuren A, van den Berkmortel F, van den Akker M, Deckx L, Buntinx F, et al. Functional status decline in older patients with breast and colorectal cancer after cancer treatment: a prospective cohort study. J Geriatr Oncol. 2017;8(3):176-84 .

38. Hayashi N, Matsuoka A, Goto H, Gotoh M, Kiyoi $\mathrm{H}$, Kodera $\mathrm{Y}$, et al. Clinical effectiveness of geriatric assessment for predicting the tolerability of outpatient chemotherapy in older adults with cancer. J Geriatr Oncol. 2018;9(1):84-6.

39. Wright JL, Parekh A, Pollock YY, Schoenborn N, Smith KL, Magnant C, et al. Use of Geriatric Assessment Tools in Selecting Therapies in Women Aged? 70 Years With Hormone Receptor-Positive Early-Stage Breast Cancer: preliminary experience with a quality improvement initiative. Int J Radiat Oncol Biol Phys. 2017;98(4):884-90.

40. Hamaker ME, Molder MT, Thielen N, van Munster BC, Schiphorst AH, van Huis LH. The effect of a geriatric evaluation on treatment decisions and outcome for older cancer patients: a systematic review. J Geriatr Oncol. 2018;9(5):430-40.
41. Puts MTE, Hardt J, Monette J, Girre V, Springall E, Alibhai SMH. Use of geriatric assessment for older adults in the oncology setting: a systematic review. J Natl Cancer Inst. 2012;104(15):1133-63.

42. Caillet P, Laurent M, Bastuji-Garin S, Liuu E, Culine S, Lagrange JL, et al. Optimal management of elderly cancer patients: usefulness of the Comprehensive Geriatric Assessment. Clin Interv Aging. 2014;9:1645-60.

43. Ramjaun A, Nassif MO, Krotneva S, Huang AR, Meguerditchian AN. Improved targeting of cancer care for older patients: a systematic review of the utility of comprehensive geriatric assessment. J Geriatr Oncol. 2013;4(3):271-81.

44. Torres CH, Hsu T. Comprehensive Geriatric Assessment in the older adult with cancer: a review. Eur Urol Focus. 2017;3(4-5):330-9.

45. Soubeyran P, Terret C, Bellera C, Bonnetain F, SaintJean $\mathrm{O}$, Galvin A, et al. Role of geriatric intervention in the treatment of older patients with cancer: rationale and design of a phase III multicenter trial. BMC Cancer. 2016;16(1):932.

46. Lund CM, Vistisen KK, Dehlendorff C, Ronholt F, Johansen JS, Nielsen DL. The effect of geriatric intervention in frail elderly patients receiving chemotherapy for colorectal cancer: a randomized trial (GERICO). BMC Cancer. 2017;17(1):1-9.

47. Driessen EJM, Janssen-Heijnen MLG, Maas HA, Dingemans AMC, van Loon JGM. Study Protocol of the NVALT25-ELDAPT Trial: selecting the optimal treatment for older patients with stage III Non-small-cell Lung Cancer. Clin Lung Cancer. 2018;19(6):e849-52. 